\title{
Measurement of vascularity as a diagnostic and prognostic tool for well differentiated thyroid tumours: comparison of different methods of assessing vascularity
}

\author{
N A C S Wong, J Willott, M J Kendall, E A Sheffield
}

\begin{abstract}
Aims-To determine whether the measurement of vascularity can be used to differentiate follicular adenomas from follicular carcinomas or to reflect the prognosis of follicular carcinomas and papillary carcinomas of the thyroid gland, and to compare four methods of assessing vascularity.

Methods-Tissue sections from 26 papillary carcinomas, 15 follicular adenomas, and 15 follicular carcinomas were stained with an antibody to CD34. A computerised image analysis system was used to calculate, for each tumour, mean endothelial areas and the mean endothelium to tumour epithelial nucleus area ratio from 10 systematically selected fields across one dimension of the tumour ("systematic field" analysis) or from the three most vascularised fields of the tumour ("hot spot" analysis). A European Organisation for Research on Treatment of Cancer (EORTC) prognostic index was calculated for each papillary carcinoma and follicular carcinoma.
\end{abstract}

Results-Significant differences in vascularity between the three tumour groups could only be shown by comparing mean endothelial area values measured from hot spots. While the hot spot median mean endothelial area of follicular carcinomas was significantly greater than that of follicular adenomas, there was a large overlap between the two groups. For follicular carcinomas, higher hot spot mean endothelial area values were related to worse prognosis as indicated by the EORTC prognostic indices. No association between vascularity and prognosis was found for the papillary carcinomas, regardless of the method of assessing vascularity.

Conclusions-Measuring endothelial area from hot spots using a computerised image analysis system is a sensitive method of assessing the vascularity of thyroid tumours. While vascularity measurement cannot be recommended as a practical tool for differentiating between malignant and benign follicular tumours, the suggestion that vascularity may reflect prognosis for follicular carcinomas deserves further study.

(f Clin Pathol 1999;52:593-597)
Keywords: thyroid tumours; vascularity; prognosis

The three commonest neoplasms of the thyroid gland are papillary carcinoma, follicular adenoma, and follicular carcinoma. ${ }^{1}$ The histological distinction between the latter two can be difficult at times. While the demonstration of capsular or vascular invasion is still regarded as the gold standard for defining a follicular carcinoma, ${ }^{1}$ there have been several attempts - as yet unsuccessful-to develop a practical tool which can reliably differentiate between benign and malignant follicular tumours. $^{2-4}$ Tumour neovascularisation is thought to play a key role in metastasis. ${ }^{5}$ It is therefore possible, in view of their metastatic potential, that follicular carcinomas are more vascular than follicular adenomas. To our knowledge, only one published study has produced data to test this hypothesis; while the investigators reported only a "marginal difference" in vascularity between follicular carcinomas and follicular adenomas, ${ }^{6}$ it is not possible to assess the significance of the finding as statistical analysis was not performed and raw data not provided.

Another potential use of tumour vascularity measurement is to reflect prognosis. Increased vascularity has been shown to correlate with worse prognosis in a variety of malignancies including breast and prostatic carcinomas and gliomas. ${ }^{7-9}$ We are aware of only two published studies of the relation between vascularity and prognosis or prognostic markers in thyroid tumours; in neither study was a significant relation shown for either papillary carcinoma or follicular carcinoma. ${ }^{1011}$

Tumour vascularity can be assessed histologically by a multitude of different techniques. Microvessel counting and the analysis of areas of most intense vascularity ("hot spots"), as was used in both the above mentioned studies, ${ }^{10}{ }^{11}$ are more vulnerable to problems with observer variation than computerised image analysis and the assessment of randomly or systematically selected fields. ${ }^{12}{ }^{13}$ Well differentiated thyroid tumours may show wide architectural variation ${ }^{1}$ and fewer tumour epithelial cells would appear to be in close contact with blood vessels in a loose papillary area than in a densely packed microfollicular area. Therefore, it may be argued that a more representative assessment of a thyroid tumour's vascularity and propensity to metastasise should involve determining the number or area 

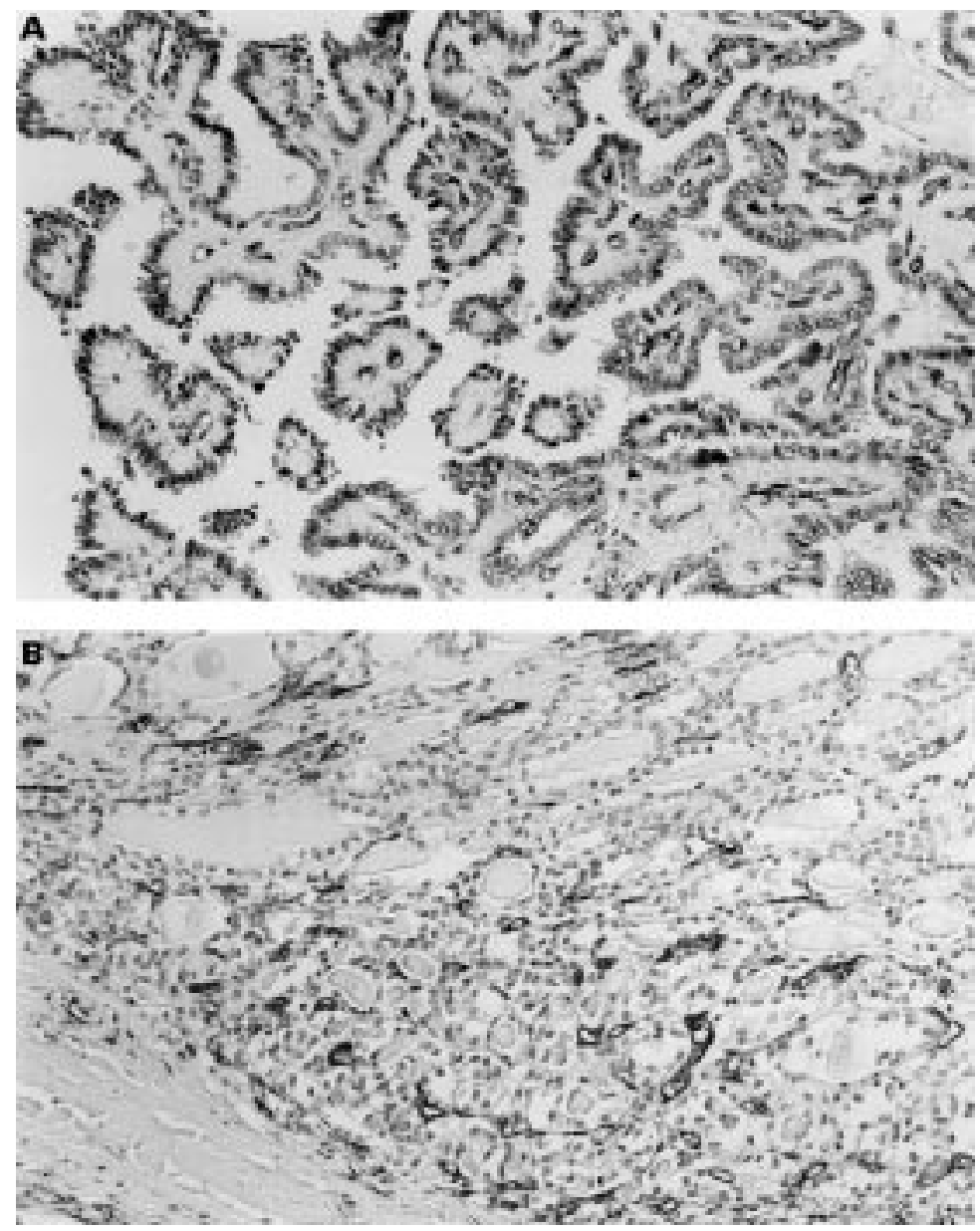

Figure 1 High power view of $(A)$ a papillary carcinoma $(\times 250$ objective $)$ and $(B)$ a follicular carcinoma ( $\times 250$ objective) showing vascular endothelium stained by an antibody to CD34. In the latter case, increased vascularity is evident at a point of capsular invasion-the fibrous capsule is seen at the bottom left corner of the figure.

of blood vessels in relation to the number or area of tumour epithelial cells in any given field.

For these reasons, it is uncertain to what extent the negative findings of previous studies of vascularity in thyroid tumours are attributable to the methods used. Our aims in the following study were therefore first, to re-examine whether measurement of vascularity may be used to distinguish follicular carcinomas from follicular adenomas, and to reflect the prognosis of follicular and papillary carcinomas; and second, to assess the extent to which different methods of assessing vascularity may influence these findings.

\section{Methods}

The study was carried out on 56 patients with tumours of the thyroid gland: 26 with papillary carcinomas (20 female, six male; median age 44 years, range 8 to 78 years), 15 with follicular adenomas (12 female, three male; median age 47 years, range 19 to 81 years), and 15 with follicular carcinomas (14 female, one male; median age 39 years, range 18 to 76 years). These patients had undergone surgical resection of their tumours between January 1985 and December 1995. The median length of follow up was 79 months (range 25 to 116 months).
A representative paraffin embedded tissue block from each of the tumours was retrieved from the archival files of the university department of pathology and microbiology at the Bristol Royal Infirmary. The tissue block bore a complete section of the tumour in most cases. Following dewaxing and rehydration, each tissue section ( $4 \mu \mathrm{m}$ thick) was stained with haematoxylin to identify epithelial nuclei, and an antibody to CD34 (QBend/10, Dako) to identify vascular endothelium (fig 1). Immunohistochemical staining for CD34 using this antibody and a peroxidase conjugated avidin method has been described previously. ${ }^{14}$

A computerised image analysis system was used to calculate the total area of endothelium and the total area of epithelial nuclei in a $\times 25$ magnified field. Images of selected fields were digitised using a three channel (RGB, all channels used) colour camera (TK-870E colour video head, JVC) linked to an IBM compatible personal computer with an image analysis computer package (Microscale TM/TS image analysis system, Digithurst). For each tumour, thresholding parameters - to allow the computer to recognise the colours representing either vascular endothelium (brown) or epithelial nuclei (blue/purple)—were set by selecting representative pixels from images of the tumour on the computer monitor. The only artefact of significance comprised lymphocyte nuclei, which could not be readily differentiated from epithelial nuclei using this image analysis system; the inability to reject this artefact and its implications for the calculated data are dealt with below (see Discussion).

For each tumour, a mean endothelial area and a mean endothelium to tumour epithelial nucleus area ratio $(\mathrm{mE} / \mathrm{N})$ were calculated using the three most vascularised fields of the tumour (hot spot analysis) or 10 systematically selected fields across the longest dimension of the tumour (systematic field analysis). The latter 10 fields were selected by determining the number of $\times 25$ magnified fields required to span the longest dimension of the tumour and thus choosing 10 equally spaced fields to span this dimension. These measurements were repeated on 10 randomly selected tumours to assess their reproducibility.

Kaplan-Meier analysis was not thought appropriate in view of the size of our study groups and the low mortality recorded over the follow up period. As a recognised surrogate indicator of prognosis, we therefore used for our analysis the European Organisation for Research on Treatment of Cancer (EORTC) prognostic index for thyroid tumours. ${ }^{15}$ An EORTC prognostic index was calculated for each follicular and papillary carcinoma using the following formula: EORTC prognostic index $=\mathrm{A}+(45 \times \mathrm{C})+(15 \times \mathrm{M})+(12 \times \mathrm{S})+$ $(10 \times \mathrm{T})+(10 \times \mathrm{D})$ where $\mathrm{A}=$ age at diagnosis in years, $C=$ anaplastic cell type $(0$ if absent, 1 if present), $M=$ distant metastasis ( 0 if none, 1 if single site, 2 if multiple sites), $\mathrm{S}=\operatorname{sex}(0$ if female, 1 if male $), \mathrm{T}=\mathrm{T}$ category ( 1 if tumour diameter $>5 \mathrm{~cm}$ or tumour fixed to adjacent structures, 0 if neither), and 
Table 1 Spearman rank correlation coefficients for the four methods of assessing vascularity studied

\begin{tabular}{llll}
\hline & $\begin{array}{l}m E A \text { and hot } \\
\text { spot analysis }\end{array}$ & $\begin{array}{l}m E A \text { and systematic } \\
\text { field analysis }\end{array}$ & $\begin{array}{l}m E / N \text { and hot } \\
\text { spot analysis }\end{array}$ \\
\hline $\mathrm{mE} / \mathrm{N}$ and systematic field analysis & 0.53 & 0.60 & 0.69 \\
$\mathrm{mE} / \mathrm{N}$ and hot spot analysis & 0.58 & 0.54 & \\
$\mathrm{mEA}$ and systematic field analysis & 0.62 & & \\
\hline
\end{tabular}

$\mathrm{mEA}$, mean endothelial area; $\mathrm{mE} / \mathrm{N}$, mean endothelial to tumour epithelial nucleus area ratio.

$\mathrm{D}=$ medullary or follicular less differentiated cancer ( 0 if absent, 1 if present).

Normal distributions cannot be assumed for the vascularity indices we studied nor for EORTC prognostic indices. Therefore, vascularity indices between any two tumour types were compared using a two tailed MannWhitney U test, while the correlations between different vascularity indices and the associations between vascularity indices and EORTC prognostic indices were studied by calculating Spearman rank correlation coefficients. All statistical analysis was performed using the computer software package SPSS v7.5.1 for Windows 95 (SPSS Inc).

\section{Results}

Of the four methods of assessing vascularity, measuring mean endothelial area from hot spots showed the least variability (differences between first and second measurements of 10 tumours: hot spot mean endothelial area analysis: median $7 \%$, range $3-13 \%$; hot spot $\mathrm{mE} / \mathrm{N}$ analysis: median $10 \%$, range $3-17 \%$; systematic field mean endothelial area analysis: median $9 \%$, range $4-15 \%$; systematic field $\mathrm{mE} / \mathrm{N}$ analysis: median $14 \%$, range $6-19 \%$ ). There were reasonable correlations between the four methods of assessing vascularity studied (table 1). For each tumour group, the mean endothelial area and $\mathrm{mE} / \mathrm{N}$ values calculated by systematic field analysis were consistently lower than those calculated from hot spots (table 2).

All three tumour types showed intratumour variation in endothelial area and $\mathrm{mE} / \mathrm{N}$. The variation in both indices was as much as 10-fold in some tumours, the highest endothelial area and $\mathrm{mE} / \mathrm{N}$ values being found in the peripheries of the tumours (data not shown). With regard to the follicular carcinomas, all the hot spot areas chosen for study were located at the periphery of the tumours. In addition, endothelial area and $\mathrm{mE} / \mathrm{N}$ values for the follicular carcinomas were particularly high around sites of capsular invasion (data not shown but see fig 1B).

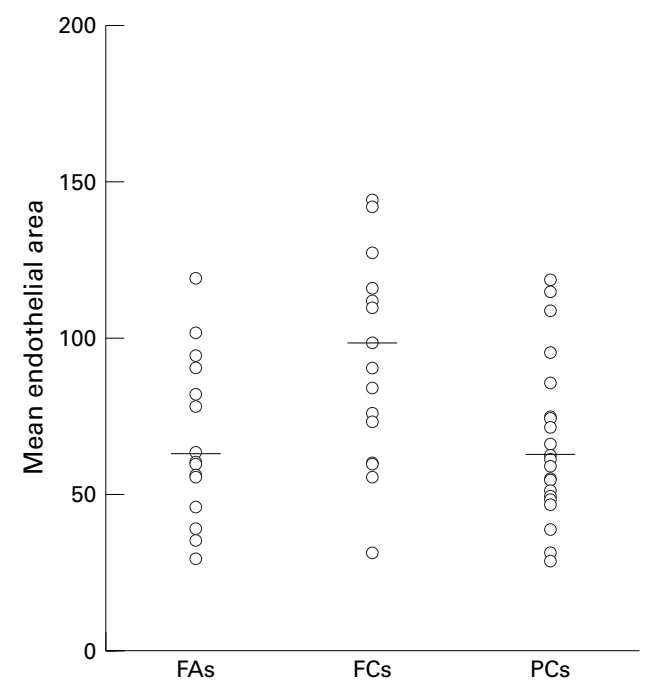

Figure 2 Hot spot mean endothelial area values (units: $\times 10^{-4} \mathrm{~mm}^{2} / \times 25$ magnified field), including median values (horizontal bars), for the three thyroid tumour groups.

By hot spot analysis, the median mean endothelial area of the follicular carcinomas was significantly greater than that of the follicular adenomas or the papillary carcinomas (fig 2, table 2). However, because the ranges of the follicular carcinomas and follicular adenomas overlapped considerably (fig 2), there was no value of mean endothelial area that, if used as a diagnostic test, would be both reasonably specific and sensitive in differentiating between the two tumour types. No significant differences between the three tumour types were found using systematic field $\mathrm{mE} / \mathrm{N}$, hot spot $\mathrm{mE} / \mathrm{N}$, or systematic field mean endothelial area analysis (table 2).

During the study follow up period, none of the papillary carcinoma patients and two of the follicular carcinoma patients died. In both the latter cases, death was directly attributable to sequelae of metastatic follicular carcinoma. There was a suggestion of a correlation between mean endothelial area and EORTC index among the follicular carcinomas, and the highest rank correlation coefficient was demonstrated using hot spot analysis (fig 3A, table 3). Using a hot spot mean endothelial area cut off value of $125 \times 10^{-4} \mathrm{~mm}^{2} / \times 25$ magnified field, two of the three follicular carcinoma patients with corresponding values above the cut off died, whereas all 12 follicular carcinoma patients with values below the cut off are currently alive ( $p=0.03$, Fisher's exact test). There was no obvious correlation between vas-

Table 2 Mean endothelial area ( $m E A)$ and mean endothelial to tumour epithelial nucleus area ratio $(m E / N)$ values by hot spot and sytematic field analyses for papillary carcinomas, follicular adenomas, and follicular carcinomas

\begin{tabular}{|c|c|c|c|c|c|}
\hline \multirow[b]{2}{*}{ Method of assessment } & \multicolumn{5}{|l|}{ Tumour type } \\
\hline & $\begin{array}{l}\text { Papillary carcinoma } \\
(n=26)\end{array}$ & $p$ Value ${ }^{*}$ & $\begin{array}{l}\text { Follicular carcinoma } \\
(n=15)\end{array}$ & $p$ Value $^{\star}$ & $\begin{array}{l}\text { Follicular adenoma } \\
(n=15)\end{array}$ \\
\hline mEA $†$ and hot spot analysis & 59.9 (28.5 to 118.2$)$ & 0.008 & 98.1 (31.5 to 143.7$)$ & 0.05 & $60.4(29.2$ to 118.8$)$ \\
\hline mEA $\dagger$ and systematic field analysis & $40.4(15.2$ to 121.8$)$ & 0.07 & $54.1(20.4$ to 105.5$)$ & 0.09 & $39.5(17.9$ to 164.7$)$ \\
\hline $\mathrm{mE} / \mathrm{N}$ and hot spot analysis & $0.40(0.10$ to 0.92$)$ & 0.49 & $0.52(0.16$ to 1.07$)$ & 0.83 & $0.42(0.19$ to 1.10$)$ \\
\hline $\mathrm{mE} / \mathrm{N}$ and systematic field analysis & $0.28(0.07$ to 0.75$)$ & 0.96 & $0.23(0.09$ to 0.59$)$ & 0.84 & $0.25(0.11$ to 0.95$)$ \\
\hline
\end{tabular}

Values are median (range).

*Significance level for comparison of two tumour types using two tailed Mann-Whitney U test.

†Units of measurement: $\times 10^{-4} \mathrm{~mm}^{2} / \times 25$ magnified field. 



Figure 3 Association between hot spot mean endothelial area values (units: $\times 10^{-4} \mathrm{~mm}^{2} / \times 25$ magnified field) and European Organisation for Research on Treatment of Cancer (EORTC) prognostic indices for $(A)$ follicular carcinomas and (B) papillary carcinomas. Patients who died are arrowed.

cularity and EORTC index among the papillary carcinomas, regardless of which type of analysis was used (table 3, fig 3B).

\section{Discussion}

We have studied the vascularity of well differentiated thyroid tumours and whether it can be used to distinguish between follicular carcinomas and follicular adenomas or reflect tumour prognosis. We used an antibody to CD34 to facilitate comparisons between our study and previous studies of vascularity and prognosis in thyroid tumours. ${ }^{11}{ }^{11}$ Further, CD34 is a more sensitive marker of vascular endothelium than factor VIII, ${ }^{12}$ as was used by Segal and colleagues in their study of follicular tumours. ${ }^{6}$ CD34 expression has also been demonstrated in haematopoietic progenitor cells and spindle cell tumours, such as dermatofibrosarcoma protuberans. ${ }^{16}$ However, such tissue and cell types are not known to exist in well differentiated thyroid tumours and

Table 3 Association between mean endothelial area $(\mathrm{mEA} A$ ) and mean endothelial to tumour epithelial nucleus area ratio $(\mathrm{mE} / \mathrm{N})$ values and European Organisation for Research on Treatment of Cancer (EORTC) prognostic indices using either hot spot or systematic field analysis, for follicular carcinomas and papillary carcinomas

\begin{tabular}{lllll}
\hline & \multicolumn{1}{c}{ Method of assessment } & & \\
\cline { 2 - 5 } & $\begin{array}{l}m E \text { A and hot spot } \\
\text { analysis }\end{array}$ & $\begin{array}{l}m E A \text { and systematic } \\
\text { field analysis }\end{array}$ & $\begin{array}{l}m E / N \text { and hot } \\
\text { spot analysis }\end{array}$ & $\begin{array}{l}m E / N \text { and } \\
\text { systematic field } \\
\text { analysis }\end{array}$ \\
\hline $\begin{array}{l}\text { Follicular } \\
\text { carcinomas }\end{array}$ & 0.34 & 0.27 & 0.17 & 0.20 \\
$\begin{array}{c}\text { Papillary } \\
\text { carcinomas }\end{array}$ & -0.11 & -0.003 & -0.15 & -0.18 \\
\hline
\end{tabular}

Values are Spearman rank correlation coefficients. therefore were unlikely to have artefactually altered our vascularity measurements.

We compared four different methods of assessing vascularity in our study. The largest differences in vascularity between different tumour types and the closest correlation with prognosis were both shown by studying only hot spots and by considering endothelial area without any adjustment for relative tumour epithelial area. Indeed, contrary to our working hypothesis, we found no evidence to suggest such an adjustment contributes to the assessment of vascularity in thyroid tumours. This apparent lack of contribution may have resulted from a weakness in our method of measuring tumour epithelial area. The computerised image analysis system used could not easily distinguish between tumour epithelial nuclei and lymphocyte nuclei, and therefore tumour nucleus area values may have been exaggerated for tumours containing large numbers of the latter cells. Nonetheless, unless a quick and simple method of specifically evaluating tumour epithelial cell area is devised, we cannot recommend incorporating this variable into the assessment of thyroid tumour vascularity.

Our results have shown that systematic field analysis is less likely to detect differences in vascularity than hot spot analysis. The reason for this appears to be that any significant differences in vascularity at tumour peripheries are negated by measurements from less vascularised areas of the tumours. Very few existing studies have compared the measurement of vascularity from hot spots to that from randomly or systematically selected fields. Goulding and colleagues used hot spot and random field analyses to study the vascularity of breast carcinomas, and found that in neither case did the correlation between vascularity and prognosis reach statistical significance. ${ }^{17}$ Unfortunately, the lack of raw data precludes further analysis to show which of these methods, if either, yielded the better correlation.

We were able to show that as a whole follicular carcinomas have significantly greater vascularity than follicular adenomas. A lack of published data in the only other study on this subject precludes an explanation of why the investigators only demonstrated a "marginal difference." The difference in vascularity between benign and malignant follicular tumours receives some support from studies of vascular endothelial growth factor (VEGF) expression by thyroid tumour cell lines. While accepted as only in vitro evidence, greater expression of this angiogenic compound was found in follicular carcinoma cell lines compared with follicular adenoma cell lines. ${ }^{18}$ Although there may be a difference in vascularity between follicular carcinomas and follicular adenomas, we found a large overlap of the range of values for the two groups. Therefore the measurement of vascularity would not have sufficiently high combined sensitivity and specificity rates to be useful as a test for differentiating between the two follicular tumour types. 
While not a prime aim of our study, we showed that the vascularity of follicular carcinomas was significantly greater than that of papillary carcinomas. This trend has also been demonstrated, though not always reaching statistical significance, in three previous studies, ${ }^{101119}$ and is once again supported by VEGF studies, where follicular carcinoma cell lines have been shown to express more VEGF than papillary carcinoma lines. ${ }^{18}$ Perhaps this difference in vascularity between tumour types explains, at least in part, the greater propensity for follicular carcinomas to metastasise by the blood stream compared with papillary carcinomas. ${ }^{1}$

There have been only two reported studies of vascularity and prognosis in thyroid tumours. Fontanini and colleagues could not show any significant relation between these two variables for follicular carcinomas $(n=25)$ or papillary carcinomas $(\mathrm{n}=57) .{ }^{10}$ In a more recent study using nine follicular carcinomas and 29 papillary carcinomas, an American group could similarly find no significant relation between vascularity and two prognostic markers - that is, locoregional metastasis and tumour recurrence. ${ }^{11}$ In the Italian study, all the three papillary carcinoma patients who died, compared with only two of the five follicular carcinoma patients who died, had tumours with microvessel counts above the median value for the respective tumour type. ${ }^{10}$ However, our findings indicate an opposite trend-that is, an association between vascularity and prognosis is suggested among follicular carcinomas but not among papillary carcinomas. We have shown a statistically significant association between high hot spot mean endothelial areas and worse prognosis for follicular carcinomas using a mean endothelial area cut off value of $125 \times 10^{-4} \mathrm{~mm}^{2} / \times 25$ magnified field. Further support for a link between higher vascularity and tumour aggressiveness comes from our own observations and from previously reported observations ${ }^{6}$ that the most vascularised areas of follicular carcinomas correspond to sites of capsular invasion. There are several possible reasons for the differences in findings between our study and the two previous studies of vascularity and prognosis in thyroid tumours. In the Italian study, ${ }^{10}$ the exact causes of death of the patients were not stated and if they were unrelated to the thyroid tumours, the study's comparative mortality analysis would be flawed. As discussed above, our findings suggest the negative results of the Italian and American studies are unlikely to relate to use of hot spot analysis without an adjustment for relative tumour epithelial area. However, both studies employed manual microvessel counting which, unlike computerised area measurement, is vulnerable to problems with observer variability and reproducibility. ${ }^{13}$ Indeed, the authors of two previous studies-one on breast carcinomas $^{20}$ and the other colorectal carcinomas $^{21}$ - were able to show a significant correlation between vascularity and prognosis by measuring vascular area but not by blood vessel counting.

\section{CONCLUSIONS}

To summarise, we have shown that, of the four different methods studied, vascularity of well differentiated thyroid tumours is best assessed by measuring endothelial area from hot spots. We cannot recommend measurement of vascularity as a practical tool for differentiating between follicular carcinomas and follicular adenomas. However, our data suggest that tumour vascularity may reflect prognosis among follicular carcinomas. A prospective study to recruit more cases of follicular carcinoma and to extend the mortality analysis of our existing cases is currently under way.

1 Rosai J. Thyroid gland. In: Rosai J, ed. Ackerman's surgical pathology, 8th ed. St Louis: CV Mosby, 1996:493-567.

2 Tuccari G, Barresi G. Immunohistochemical demonstration of ceruloplasmin in follicular adenomas and thyroid carcinomas. Histopathology 1987;11:723-31.

3 Vierbuchen M, Schröder S, Uhlenbruck G, et al. CA 50 and CA 19-9 antigen expression in normal, hyperplastic and neoplastic thyroid tissue. Lab Invest 1989;60:726-32.

4 Hruban RH, Huvos AG, Traganos F, et al. Follicular neoplasms of the thyroid in men older than 50 years of age. A DNA flow cytometric study. Am 7 Clin Pathol 1990;94:527-32.

5 Jiang WG, Puntis MCA, Hallett MB. Molecular and cellular basis of cancer invasion and metastasis: implications for treatment. Br f Cancer 1994;81:1576-90.

6 Segal K, Shpitzer T, Feinmesser M, et al. Angiogenesis in follicular tumours of the thyroid. F Surg Oncol 1996;63:958 .

7 Fox SB, Leek RD, Weekes MP, et al. Quantitation and prognostic value of breast cancer angiogenesis: comparison of microvessel density, Chalkley count, and computer image analysis. F Pathol 1995;177:275-83.

8 Vesalainen S, Lipponen P, Talja M, et al. Tumour vascularity and basement membrane structure as prognostic factors in T1-2, M0 prostatic adenocarcinoma. Anticancer Res 1994;14:709-14

9 Leon SP, Folkerth RD, Black PMcL. Microvessel density is a prognostic indicator for patients with astroglial brain tumours. Cancer 1996;77:362-72.

10 Fontanini G, Vignati S, Pacini F, et al. Microvessel count: an indicator of poor outcome in medullary carcinoma but not other types of thyroid carcinoma. Mod Pathol 1996;9:63641 .

11 Goldenberg JD, Portugal LG, Wenig BL, et al. Welldifferentiated thyroid tumours: p53 mutation status and differentiated thyroid tumours: p53 mutation
microvessel density. Head Neck 1998;20:152-8.

12 Fox SB. Tumour angiogenesis and prognosis. Histopathology 1997;30:294-301

13 Barbareschi M, Gasparini G, Morelli L, et al. Novel methods for the determination of the angiogenic activity of human tumors. Breast Cancer Res Treat 1995;36:181-92.

14 Ramani P, Bradley NJ, Fletcher CDM. QBEND/10, a new monoclonal antibody to endothelium: assessment of its diagnostic utility in paraffin sections. Histopathology 1990; 17:237-42.

15 Byar DD, Green SB, Dor P, et al. A prognostic index for thyroid carcinoma: a study of the EORTC thyroid cancer cooperative group. Eur f Cancer 1979;15:1033-41.

16 Cohen PR, Rapini RP, Farhood AI. Expression of the human hematopoietic progenitor cell antigen CD34 in vascular and spindle cell tumours. F Cutan Pathol 1993;20:1520 .

17 Goulding H, Nik Abdul Rashid NF, Robertson JF, et al. Assessment of angiogenesis in breast carcinoma: an imporAssessment of angiogenesis in breast carcinoma: an impor-

tant factor in prognosis? Hum Pathol 1995;26:1196-200.
18 Viglietto G, Maglione D, Rambaldi M, et al. Upregulation of vascular endothelial growth factor (VEGF) and downregulation of placenta growth factor (PIGF) associated with malignancy in human thyroid tumors and cell lines. Oncogene 1995;11:1569-79.

9 Herrmann G, Schumm-Draeger P-M, Müller C, et al. T lymphocytes, CD68-positive cells and vascularisation in thyroid carcinomas. F Cancer Res Clin Oncol 1994;120:6516.

20 Simpson JF, Ann C, Battifora $\mathrm{H}$, et al. Vascular surface area a prognostic indicator in invasive breast cancer [abstract]. Mod Pathol 1994;7:22a.

21 Pavlopoulos PM, Konstantinidou AE, Agapitos E, et al. A morphometric study of neovascularisation in colorectal carcinoma. Cancer 1998;83:2067-75. 